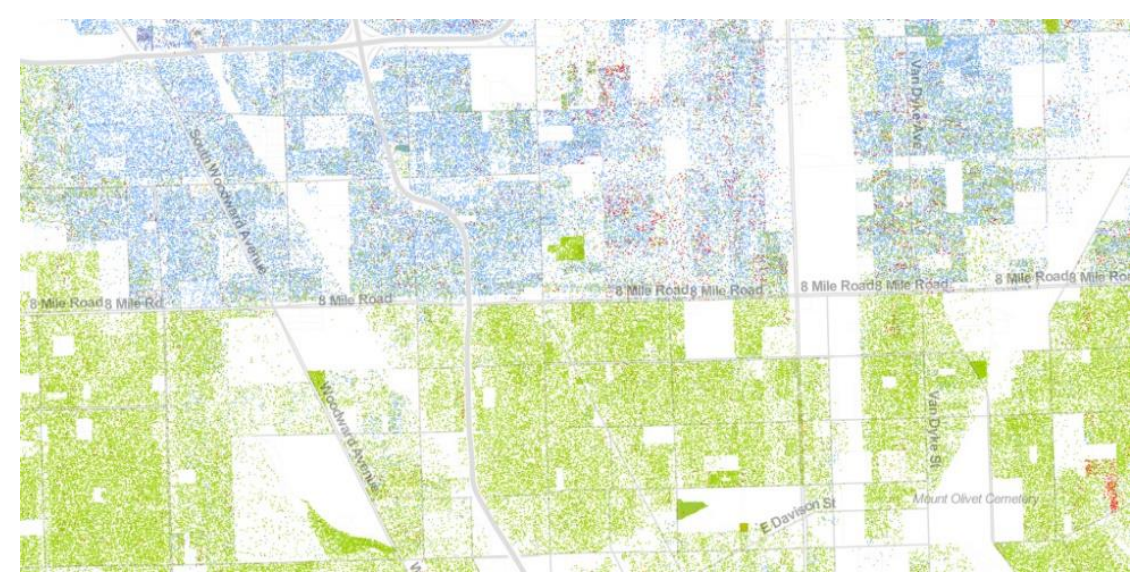

\title{
Por uma etnografia das redes sem fio comunitárias: implementar tecnologias descentralizadas para agir contra a crise em Detroit $^{1}$
}

\author{
For an ethnography of community wireless networks: Implementing decentralized \\ technologies to act against the Detroit crisis. \\ François Huguet ${ }^{2}$ \\ Tradução de Guilherme Adorno ${ }^{3}$
}

\section{Resumo:}

As relações urbanas são atravessadas por um embate entre a organização administrativa do espaço pelos órgãos oficiais e as práticas cotidianas que buscam alternativas frente ao poder do Estado. Neste artigo, é analisado o modo como esse embate produz estratégias e práticas econômicas alternativas na cidade americana de Detroit para escapar da chamada "crise urbana" no Capitalismo contemporâneo. São descritas outras formas de pensar um modelo urbano, concebido e apoiado pela participação dos cidadãos e seu "poder de agir" mediante o uso de objetos tecnológicos. A análise de entrevistas com os sujeitos destas diferentes práticas aponta para uma vontade de reconexão, buscando engendrar uma dinâmica da mudança social na relação com as infraestruturas tecnológicas.

Palavras-chave: etnografia; tecnologia; cidade; redes sem fio.
Abstract:
The urban relations are crossed by a clash between the administrative organization of space by official agencies and daily practices that seek alternatives to the power of the state. This article analyze how this struggle produces strategies and alternative economic practices in the American city of Detroit to escape the so-called "urban crisis" in contemporary capitalism. We describe different ways of thinking an urban model, designed and supported by the participation of citizens and their "power to act" through the use of technological objects. The analysis of interviews with the subjects of these different practices points to a desire to reconnect, seeking to engender a dynamic of social change in relation to the technological infrastructure.
Keywords: ethnography; technology; city; wireless networks

\footnotetext{
${ }^{1}$ Tradução do texto original "Pour une ethnographie des réseaux sans fil communautaires: Implémenter es technologies décentralisées pour agir contre la crise à Détroit."

${ }^{2} \mathrm{PhD}$ in Media Studies pela Telecom ParisTech, endereço: 7, rue de 1'Asile Popincourt, 75011 - Paris. Email: huguet.f@gmail.com

${ }^{3}$ Doutor em Linguística, pelo IEL - UNICAMP. E-mail: guiadorno1@gmail.com
} 


\section{Cidade selvagem VS. Cidade selvagem do capitalismo mundializado}

18 de julho de 2013, a cidade americana de Detroit se declarava oficialmente em falência e apresentava publicamente os números vertiginosos de sua dívida: 18,5 bilhões de dólares ${ }^{5}$. Desde então, os desfiles de estatísticas fazem crer em um lugar de apocalipse e de abandono. A cidade foi colocada sob o jugo de um administrador judiciário e o governo republicano do Estado de Michigan exerce, atualmente, um peso político-econômico massivo sobre a cidade democrata que é Detroit. As instituições municipais devem prestar contas a uma entidade nomeada pelo governador federal Rick Synder em março de 2013 e são despossuídas da maior parte de suas competências. Motor City, antiga menina dos olhos industriais do Midwest americano, agora é uma pálida figura e parece ser um lugar improvável para qualquer recuperação. Passada de 1 849568 habitantes em 1950 para 713777 em 20106, Detroit ilustra um nome importante de fenômenos urbanos característicos de certas localidades americanas: shrinking cities, white flight, gentrificaçao, enfraquecimento progressivo das cidadescentro, formação de guetos, segregação socioespacial ${ }^{7}$, etc.

\footnotetext{
${ }^{4}$ Do latim: "Nós esperamos tempos melhores, elas renascerá de suas cinzas"; lema de Detroit (Michigan, États-Unis) inscrito sobre a bandeira da cidade depois do incêndio que devastou a maior parte da cidade em 1805.

5 Le Monde com AFP [18 de julho de 2013], "A cidade americana de Detroit declara falência" [http://www.lemonde.fr/ameriques/article/2013/07/18/la-ville-americaine-de-detroit-se-declare-enfaillite 3449873 3222.html].

${ }^{6}$ Dados do Brookings, Metropolitan Program, Allan Mallach, depois do Census American Community Survey, 2010 [http://www.census.gov/acs/www/].

${ }^{7}$ Sobre este ponto, ver VANHEMERT, K. [26 de Agosto de 2013], Wired, « The Best Map Ever Made of America's Racial Segregation » [http://www.wired.com/design/2013/08/how-segregated-is-your-citythis-eye-opening-map-shows-you\#slideid-210281].
} 


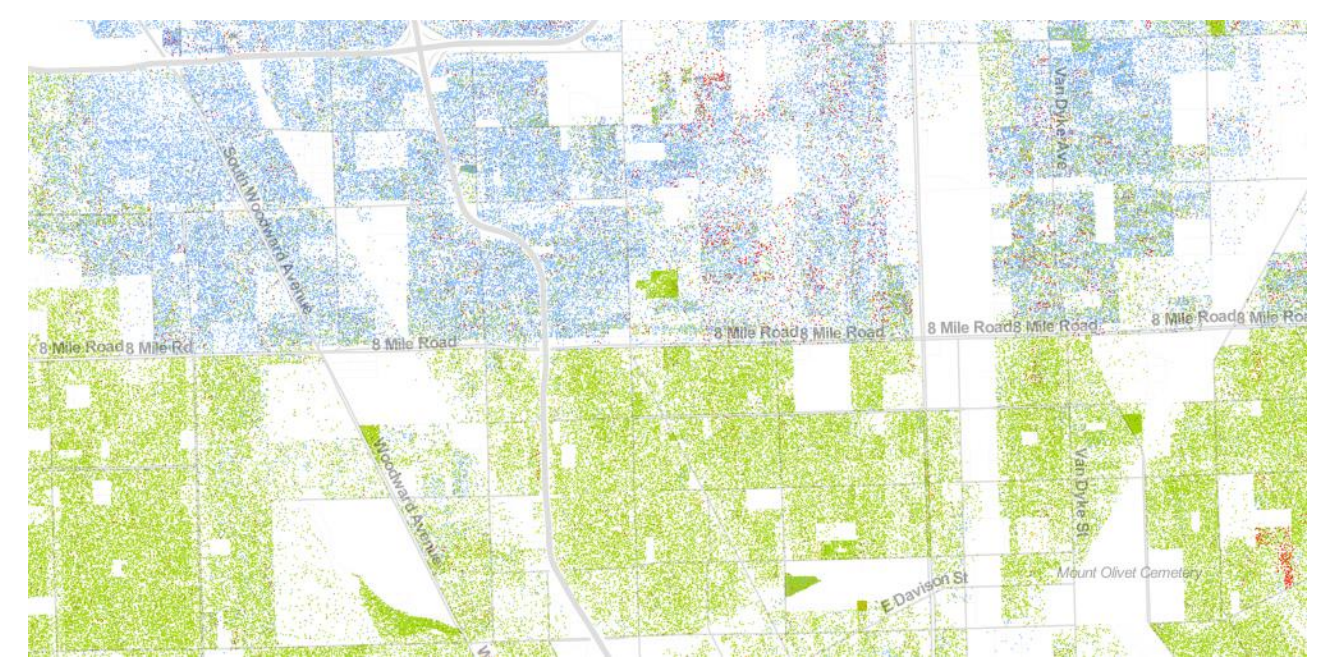

Dustin Cable - Weldon Cooper Center for Public Service - University of Virginia. Cartografia da cidade de Detroit e visualização das origens étnicas de seus habitantes: pontos azuis = população branca; pontos verdes: população afro-americana; pontos vermelhos $=$ asiáticos. A delimitação que se observa aqui corresponde a 8 Mile Road, fronteira administrativa da municipalidade e começo das periferias ricas que cercam a cidade.Extraído de VANHEMERT, K. [26 de Agosto de 2013], The Best Map Ever Made of America's Racial Segregation.

\section{Comparing Detroit to three other major cities}

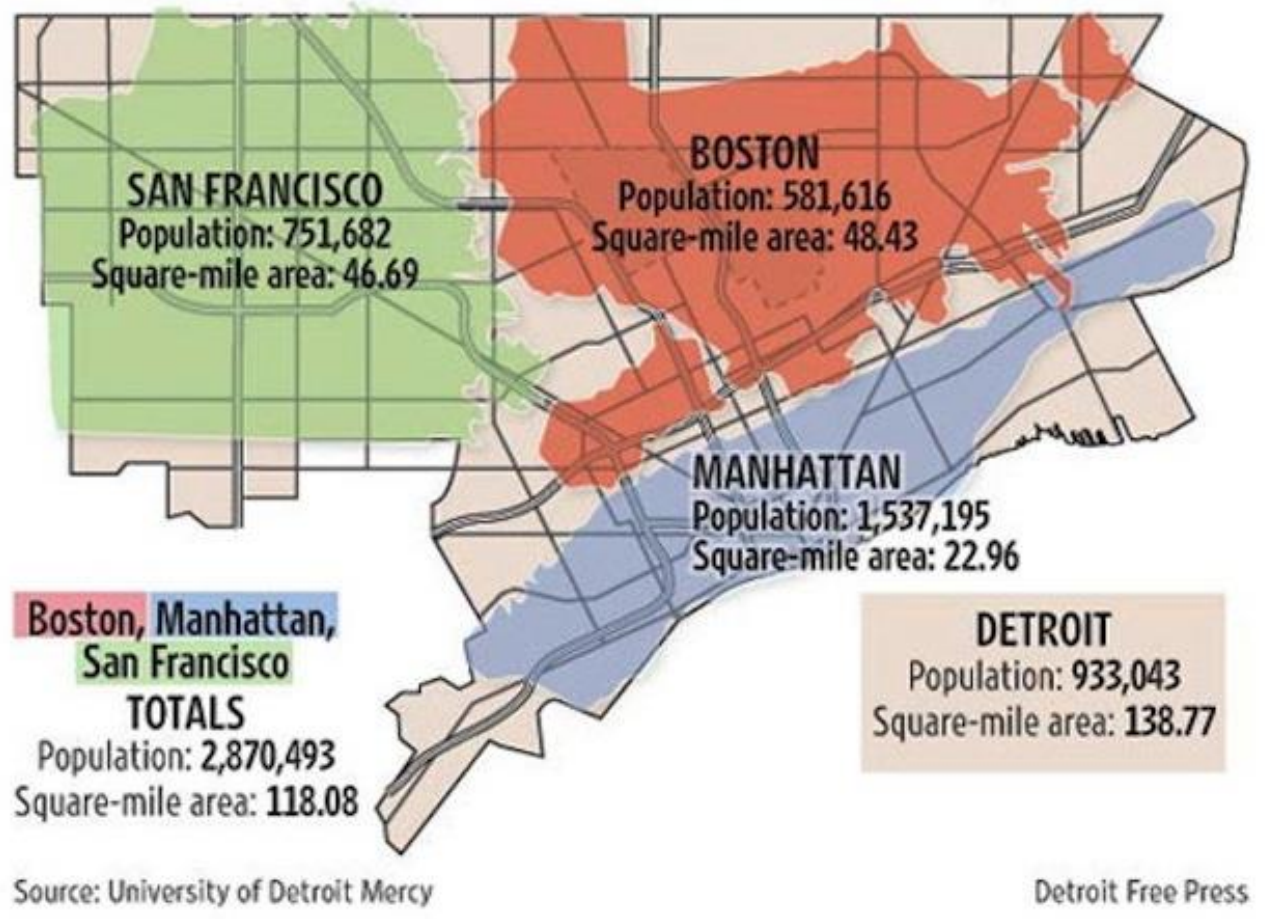

Detroit Free Press - University of Detroit Mercy. Extraído de STAES, J.A. [12 de Agosto de 2012], Comparing Detroit To Other Cities? Look At The Map!

Combinada a uma gestão de gastos públicos calamitosa, ao colapso da indústria automotiva e a uma corrupção há muito tempo instalada alto escalão do poder municipal, a imagem da cidade parece colar perfeitamente à polissemia da palavra "crise". Em Detroit, esta última é, a um só tempo, econômica, política, social e 
sistêmica. No entanto, no seio dessa situação que não se explica unicamente pelos fenômenos evocados anteriormente ${ }^{8}$, mas, sobretudo, pelos mais de sessenta anos de declínio socioeconômico de um modo de cidade especial (SUGRUE, 1996), Detroit oculta um número importante de estratégias e de práticas econômicas alternativas que correspondem à "adaptação espontânea dos modos de vida das pessoas aos constrangimentos e às oportunidades decorrentes da crise" (CASTELLS, 2012). Mais do que outras metrópoles operárias negras americanas (como Baltimore), Detroit conta com numerosas iniciativas econômicas, culturais e sociotécnicas que demandam igualmente meios para se desvencilhar da situação de crise urbana (GALLAGHER, 2013). A Paris do Midwest americano é efetivamente hoje reputada por sua atitude "faça você mesmo" ("Do It Yourself”) e suas iniciativas de agricultura urbana em grande escala. Mas esses projetos socioagrículas se atritam com outras iniciativas como os bancos alimentares cooperativos ou ainda projetos de associação não lucrativos que reinventam o que nós designamos na França por Educação Popular e que os atores desses grupos nomeiam "community organizing" ou "transformative work". Depois de seguidos anos gloriosos do fordismo, desde 1960, os estragos da desindustrialização, os cidadãos de Detroit parecem depender hoje de sua própria engenhosidade para sustentarem suas necessidades.

Esse movimento prefigura, segundo nós, uma via interessante para estudar quando se aborda a noção de crise e de resiliência urbana, ${ }^{9}$ uma economia local pósindustrial fundada sobre a ajuda mútua, a partilha, mas, também, e, sobretudo, sobre uma nova relação com as infraestruturas públicas e privadas (telecomunicação, transportes, educação, gestão do lixo, etc.). A cidade comporta igualmente um número importante de acontecimentos, de práticas particulares e de lugares dedicados à inovação digital. Um dos eixos de desenvolvimento de sua política de revitalização urbana concerne, de toda maneira, à economia digital e às indústrias criativas ${ }^{10}$.

\footnotetext{
${ }^{8}$ Sobre este ponto, ver KLEINE, R. [4 de Agosto 2013], Detroit Free Press, "How the State of Michigan helped bankrupt Detroit" [http://www.freep.com/article/20130804/OPINION05/308040048/detroitfinancial-crisis-state-of-michigan-causes-bankruptcy].

${ }^{9}$ Capacidade de uma cidade de lidar com um trauma, de enfrenta-lo e a buscar os meios para se reconstruir.

10 Sobre este ponto, ver 2012 Detroit Strategic Framework Plan, Detroit Future City [http://www.detroitlongterm.com].
} 
No centro de todas essas iniciativas ao mesmo tempo cívicas, técnicoeconômicas e socioculturais, uma ideia fixa: construir modelos, projetos e infraestruturas ${ }^{11}$ horizontais (EGYEDI \& MEHOS, 2012), autônomos e decentralizados; pensar um modelo urbano diferente, concebido e apoiado pela participação dos cidadãos e seu “poder de agir” (PROULX, 2012).

Apoiando-se sobre a análise de certos aspectos dessas práticas econômicas alternativas, nossa pesquisa visa compreender de qual maneira uma forma de infraestrutura de comunicação local e autônoma, as redes MESH no caso (ou redes comunitárias sem fio ${ }^{12}$ ), ilustra a ligação entre crise econômica e surgimento ou reforço de práticas implementadas pela sociedade civil de Detroit. Nesse quadro, nós pesquisamos sobre as comunidades que colocam em cena esse gênero de redes locais, sobre suas dinâmicas, suas ambições, seus diferentes projetos e, desse fato, sobre o surgimento de uma forma de mídia especial (GENTÈS, 2013), cujos aspectos políticos são reivindicados intrinsecamente. Este artigo apresentará extratos de entrevistas realizadas com diferentes pessoas engajadas nos desenvolvimentos dessas redes comunitárias sem fio. Nós apresentaremos aqui extratos de entrevistas sob uma forma suficientemente "bruta" em que a noção de "crise" é abordada e onde meios e/ou práticas de escapar a esse conceito são mencionadas (entrevistas realizadas entre maio e julho de 2013). Trata-se de extratos de um "caderno etnográfico" em que se misturam testemunhos e análises breves no qual nós descrevemos o mais simplesmente possível a historia e o ecossistema dessas redes sem fio e desses diferentes atores. Trata-se, aqui, de dar a ver uma "fotografia" de certo projeto tecnomidiáticos próprios a esta cidade em crise. De uma maneira mais global, nossa pesquisa visa mostrar como essas iniciativas trazem à luz questionamentos relativos à materialidade das redes de comunicação existente e aos papeis que jogam os usuários na organização e na manutenção de uma rede social digital (PROULX, 2012), de uma infraestrutura de comunicação. Nossa

\footnotetext{
${ }^{11}$ Infraestruturas de telecomunicações, agrícolas, pedagógicas, gestão do lixo, etc. Cf. EGYEDI, T. M. and MEHOS, D. C. (2012), Inverse infrastructures. Disrupting Networks from Below, Edward Elgar, Northampton.

12 Metro Scale Ad-Hoc Network, protocolo de roteamento e de estabelecimento de redes locais sem fio decentralizadas em que cada nó de rede torna-se retransmissor para os outros pares ("peer-to-peer" móvel, redes de antenas autônomas e/ou aparelhos de comunicação como os computadores, smartphones, roteadores Wi-fi, etc). A rede também estabelecida não comporta então um ponto central e é assim mais resistente aos cortes e/ou aos controles exteriores. Poderíamos comparar esse tipo de infraestruturas de comunicação a uma rede de usuários conectados entre eles via ondas de radio de intervalos cursos (um tipo de rede bluetooth expandida). Esta tecnologia de origem militar permite implantar uma rede de comunicação robusta e independente entre computadores, telefones ou terminais de comunicação, em circuito fechado, ou conectado ao mesmo em um ponto da rede Internet ou GSM.
} 
pesquisa visa igualmente compreender de que maneiras esses discursos sobre as redes de telecomunicações decentralizadas e autônomas apreendem a noção de co-construção em torno de debates e do que Bernard Stiegles chama a era econômica da contribuição (Stiegler, 2008). Fora dos termos de "crise", de "catástrofe" e de "falência", este artigo quer apresentar projetos inovadores e certos detalhes do contexto no qual essas práticas alternativas se desenvolvem. Ele deseja restituir uma fala rica e complexa, aquela de grupos de cidadãos, pivôs locais da participação cívica e que tendem a ser considerados por alguns como os atores da revitalização urbana americana ${ }^{13}$. Comunidades de cidadãos que se apoiam doravante sobre as Novas Tecnologias da Informação e da Comunicação (NTIC) para pensar não mais um modelo urbano Top-Downs (vertical, pensar de cima para baixo), mas uma cidade horizontal; irredutíveis grupos de indivíduos que concebem Motor City não mais uma "cidade selvagem", mas como a "cidade selvagem do capitalismo mundializado"14 que sofre, há muitos anos, as consequências do colapso de uma indústria orientada somente para o automóvel, procrastinações de um capitalismo destrutivo e de demasiados comentadores mal informados.

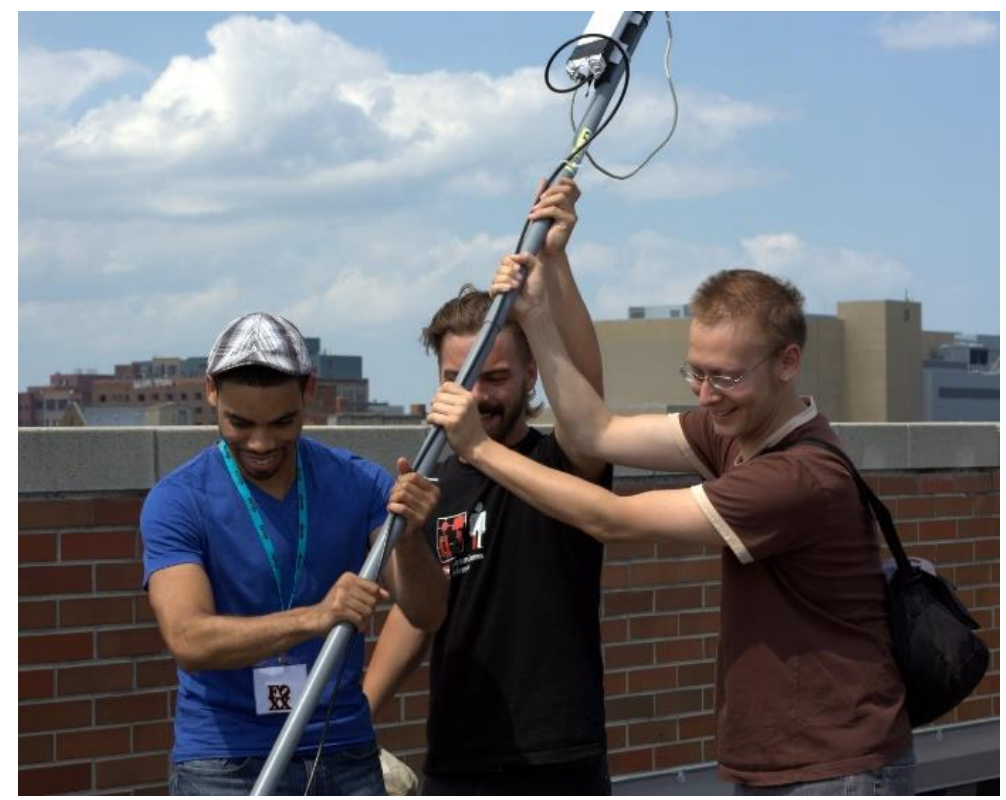

Preston Rhea - Licence CC BY-SA 2.0. Montagem de um roteador (antena) MESH-Wi-Fi instalado sobre o telhado de um bairro de MidTown em Detroit, junho de 2011.

\footnotetext{
${ }^{13}$ Sobre este ponto, ver COSME, C. (2012), Politique de la ville, l'expérience américaine, Centre d'analyse stratégique, la documentation Française, Paris.

${ }^{14}$ Sobre este ponto, ver GOËTA, S. [1 de junho de 2012], UrbaNews.fr, "Détroit n'est pas une silencieuse ville sauvage. [La critique, par Aaron Handelsman] [http://www.urbanews.fr/2012/06/01/21179-detroit-nest-pas-une-silencieuse-ville-sauvage-la-critiquepar-aaron-handelsman/\#.UioDgLwUVTU].
} 


\section{Problemáticas e contexto das conexões "MESH"}

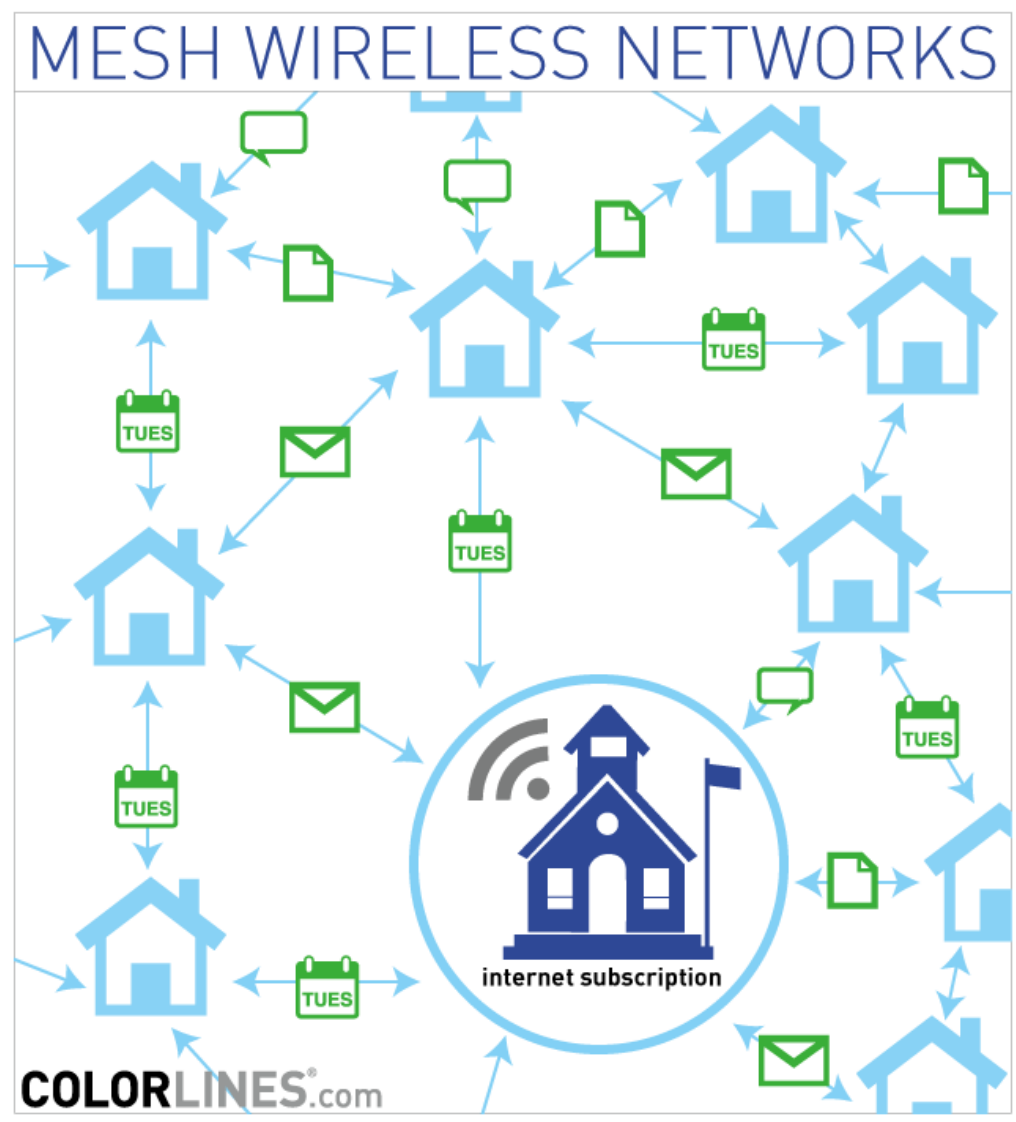

ColorLines.com - A Tech Innovation.

In: Detroit: Connect People, Not Computers (setembro de 2012)

O estabelecimento de redes MESH (Metro Scale Ad-hoc Network) não é algo recente. Esse roteamento (MANET - Mobile Ad NETwork, desenvolvido no fim dos anos 1990) é originário da pesquisa militar. Ele permite implantar uma rede de comunicação robusta e independente entre computadores, telefones ou todo outro terminal de comunicação em circuito fechado e/ou conectado ao menos em um ponto a uma rede Internet e/ou GSM. Essa rede é feita em malhas, o que quer dizer que cada um de seus nós pode servir de retransmissão, de ponto de ligação aos outros nós do conjunto que comunica via ondas de rádio Wi-fi. Assim, ele pode se reconfigurar em permanência e estabelecer comunicações dinâmicas entre pontos mais ou menos móveis (um tipo de rede Bluetooth multi-utilizadores). Até 2011, esse tipo de rede servia, sobretudo, para fazer comunicar sensores entre eles ou ainda para otimizar zonas de cobertura Wi-Fi (aeroportos, por exemplo) reduzindo custos de instalação de cabos, modens, etc. Em 2011, depois das insurreições árabes, esta tecnologia apareceu, então, como uma solução para casos de censura e de corte de telecomunicações por regimes autoritários: a aparelhagem móvel dos manifestantes, compostas de smartphones, 
tabletes, computadores, assim como pequenas antenas localizadas sobre telhados, podiam tornar-se infraestrutura de comunicação. Se cada objeto comunicante podia se conectar ao menos a um outro objeto em co-presença, era possível criar uma rede em malhas independente, autônoma e movente quase impossível de cortar ou de censurar sendo dado seu caráter espontâneo, local e decentralizado (não dependendo de um servidor informático central, de uma infraestrutura global como as emblemáticas redes sociais Facebook e Twitter). L'Open Technology Initiative (OTI), grupo de trabalho da New America Foundation ${ }^{15}$, think-tank instalada em Washington DC, apresentou nesse momento um programa de rede (network) MESH multi-plataforma batizado Commotion $^{16}$ (COMmunity Open Technology Information Online Network). Batizado em um primeiro momento como "Internet in a Suitcase", esse projeto recebeu, em 2011, uma ajuda consequente do departamento do Estado americano. Essas duas principais zonas de teste e de desenvolvimento são Detroit e Nova York (mais precisamente o bairro isolado de Red Hook no Brooklyn). Esta tecnologia não foi desenvolvida em prioridade no Oriente Médio, mas antes nas cidades em "crise" nos Estados Unidos (segundos seus criadores, ela não era "estável” o suficiente para não ser utilizada sem o conhecimento dos militantes liberais árabes).

Em Detroit, a Open Technology Initiative (OTI) trabalha conjuntamente com Allied Media Project ${ }^{17}$, associação sem fins lucrativos, organizadora da Allied Media Conference e portadora de um número importante de projetos abrangendo desde a educação até tecnologias digitais (vídeo, som, grafismo, programação eletrônica, etc.) com o treinamento de Digital Justice e uma estratégia de revitalização urbana horizontal. Esses Digital Stewards, tipos de mediadores, gestores socioculturais voluntários, fazem cursos semanais durante seis meses e desenvolvem um projeto de revitalização socioeconômica de seus bairros mediante as tecnologias digitais. Para integrar esta formação, eles devem apresentar um projeto de desenvolvimento que será aprofundado e apoiado pela perícia de diversos interessados. Nós acompanhamos três desses grupos (promoção 2013) que decidiram implementar redes MESH nos bairros onde eles residiam e com diferentes objetivos. Um desses grupos, o do projeto ECOSphere, baseado no nordeste da cidade e conduzido por três membros da

\footnotetext{
${ }^{15} \mathrm{http}$ ://oti.newamerica.net.

16 Sobre este ponto, ver GLANZ, J. \& MARKOFF, J. [12 de junho 2011], The New York Times, "U.S. Underwrites Internet Detour Around Censors" http://www.nytimes.com/2011/06/12/world/12internet html?pagewanted=all].

${ }^{17}$ http://alliedmedia.org \& http://amc.alliedmedia.org .
} 
associação Modern Evolution, considera esse projeto de uma maneira bem particular. Os extratos que nós apresentamos neste artigo são todos vindos de entrevistas que nós realizamos com essas três pessoas. Para elas, criar uma rede MESH é um pretexto que permite vislumbrar uma nova forma de ecossistema econômico para a cidade de Detroit, um meio de sair da crise dessa cidade que eles nomeiam com suas próprias palavras como "Ground zero".

Entrevistado: Eu dizia que, na Modern Evolution, nosso trabalho consiste em construir pontes entre todas as iniciativas que já existem, em duplicá-las, replicá-la [...] Antes de falar de viabilidade econômica, nós precisamos de um ecossistema digital. É por isto que nós começamos pelo Wi-Fi. As pessoas devem estar conectadas. É a nova economia [...] e a chave é o acesso.

[...] Nós queremos fazer uma comunidade sem fio tudo em conjunto [...] desde então, nós levamos o modelo decentralizado para as pessoas.

[...] Desde então o Wi-fi está por todo lugar exceto onde? Nos bairros marginalizados, você vê o que eu quero dizer?

Nesses primeiros extratos, não é uma questão de aplicações ou de serviços especiais suportados pela rede comunitária sem fio criada a partir do treinamento (se não é fornecido um acesso à Internet através de sua infraestrutura de comunicação, então através de sua rede MESH). Para o grupo de Modern Evolution, estabelecer uma rede comunitária é satisfazer a necessidade de conexão do seu bairro, que é muito pouco equipado em acesso à Internet, é compartilhar esta última com outras pessoas, outros habitantes do bairro, da quadra (longe de Midtown, zona de revitalização urbana prioritária). No entanto, contrariamente ao East Side da cidade, pouco habitada e composta majoritariamente de terrenos vazios e de casas abandonadas, o bairro onde se desenvolve o projeto batizado como ECOSphere (North West Side) possui uma densidade de habitação razoavelmente importante. Trata-se de antigos loteamentos dos supervisores das fábricas automotivas Ford. Apesar dessa densidade, existe pouco acesso à Internet, pois os custos de instalação e de funcionamento de uma conexão são muito elevados. A solução que eles tentam implementar busca contornar esses custos elevados e mostrar que um modelo de partilha pode se pensar também sobre outros domínios e outros níveis (segurança, transporte, educação, reciclagem, sistema de praças alimentares, etc.), que faz parte dos componentes de uma alavanca de desenvolvimento socioeconômico original: 
Entrevistado: [...] Nós pensávamos que se tratava de uma ideia genial: começar a juntar as pessoas como um angariador de fundos simpático. É preciso começar a reunir, de maneira que nós possamos pagar nossa fatura de Internet juntos. E, se fazemos isso com a fatura da Internet, nós poderíamos, talvez, fazer com nossa fatura de eletricidade? Você vê, isso pode despertar o interesse para esse gênero de soluções de financiamento criativas. De fato, nós tocamos aqui à principal problemática de nosso projeto: a ideologia.

[...]

Se pudéssemos instalar antenas utilizando a energia solar ou outras formas de sistemas energéticos, não teríamos que utilizar a eletricidade de um terceiro e seríamos independentes. Com o MESH, a única coisa que precisaríamos é a eletricidade para interligar a antena com outro roteador, conectado à rede.

[...]

Johns se ocupa de questões energéticas, Heru de questões tecnológicas, eu me ocupo dos financiamentos. E nossa sinergia vem daí. Nós nos ajudamos uns aos outros, e graça a isto, soluções interessantes surgem.

Criar uma rede comunitária sem fio pode aparecer, então, como um pretexto para engajar pessoas para formas de ação cívicas e sociopolíticas que concernem às infraestruturas e os serviços da cidade (que estão em estado deploráveis: polícia e bombeiros com efetivos reduzidos, rede de transportes e sistemas elétricos falhando, deficiente coleta de lixo, etc). Essas redes, vindas dos treinamentos realizados por Allied Media e OTI, são os elementos constitutivos de um programa de Digital Stewardship, de cidadãos retransmissores (mediadores em tecnologia digital) para os bairros marginalizados de uma cidade especial, lugar de colapso da American way of life. Mas, poderíamos também ver no desenvolvimento dessas tecnologias uma prática econômica alternativa ao serviço de um tipo de resiliência urbana. De fato, conjugados às outras práticas alternativas (notadamente agrícolas), esses treinamentos e esses projetos de infraestrutura e de participação ativa de cidadãos tornam-se meios de cicatrização citadina, repostas ao vazio deixado pela municipalidade em falência e também oportunidades para as organizações comunitárias sem fins lucrativos de testarem modelos urbanos alternativos. Nessa "crise", dessas cinzas, e do lugar que os membros do projeto ECOSphere - Modern Evolution chamam eles mesmos de "Ground zero", deveria poder emergir uma nova relação com a cidade; uma resiliência de uma só vez cívica e tecnológica que refundaria a qualidade de cidade de Detroit e colocaria as primeiras pedras de uma cidade mais cooperativa, onde participação e engajamento do 
cidadão seriam mais importantes. As infraestruturas de comunicação aqui discutidas visam construir um modelo cívico desse tipo e conscientizar sobre os comportamentos que ele exige.

A noção de Digital Justice defendida por Allied Media Projects, vindas ao mesmo tempo do pensamento de Grace Lee Boggs ${ }^{18}$, de Paulo Freire e de Saul Alinsky ${ }^{19}$, integra-se nesta ideia de resiliência, de cicatrização urbana. Essa justiça digital consiste em defender quatro pilares característicos nos projetos implementados, mas, também, e, sobretudo, a noção de direito fundamental à comunicação (em outros termos, combater a fratura digital). Esses quatro pilares são: o direito ao acesso (à Internet, às NTIC, às mídias de maneira geral), a participação, a partilha e a cooperação no seio de grupo de cidadão que eles chamam de "communities" (habitantes do mesmo bairro e/ou pessoas reunidas em torno de um interesse comum). Para as pessoas que nós entrevistamos, a ideia de reconstruir a vida cotidiana e as práticas cidadãs em torno de práticas autônomas, que não repousam sobre as instituições financeiras e/ou formas de governo público, tornou-se mais realista que o modelo tradicional de comportamento socioeconômico. As redes MESH são um passo em frente para essa ideia de se organizar em comunidade em torno de um interesse comum, nesse caso relatado aqui, reduzir o custo do acesso à Internet e, assim, a fatura digital, para aumentar a participação cidadão e o engajamento de cada um.

Entrevistado: Aqui, é o maro zero da América, verdadeiramente. Você sabe, essa região era uma das mais prósperas dos Estados Unidos em uma época e, agora, é uma das mais pobres. O que aconteceu? Trata-se também de uma história de inovação e revolução em termos de comunidades, de social; quando olhamos a história, um número importante de pessoas engajadas veio dessa região, que sempre foi um antro composto de operários [...]

Somente da comunidade afro-americana, as três iniciativas mais 'visiveis' vieram aqui. A luta por Direitos cívicos, The Nation of Islam nasceu aqui, a Republic of New Africa nasceu aqui e os Black Christian Nationalists nasceram aqui.

[...]

Agora, e se você vai nessas fazendas urbanas, você não verá somente uma "raça" ou um único grupo de idade. Há jovens, negros, brancos, asiáticos, todos trabalham juntos nessa lógica de parceria inovadora, [...] é algo hoje, não um blábláblá, não é um think tank, aqui é um laboratório para ação.

\footnotetext{
${ }^{18}$ Ativista e filósofo originário de Detroit, militante do Black Power nos anos 60.

${ }^{19}$ Militante e sociólogo americano originário de Chicago, geralmente reconhecido como ai fundado da "community organizing".
} 


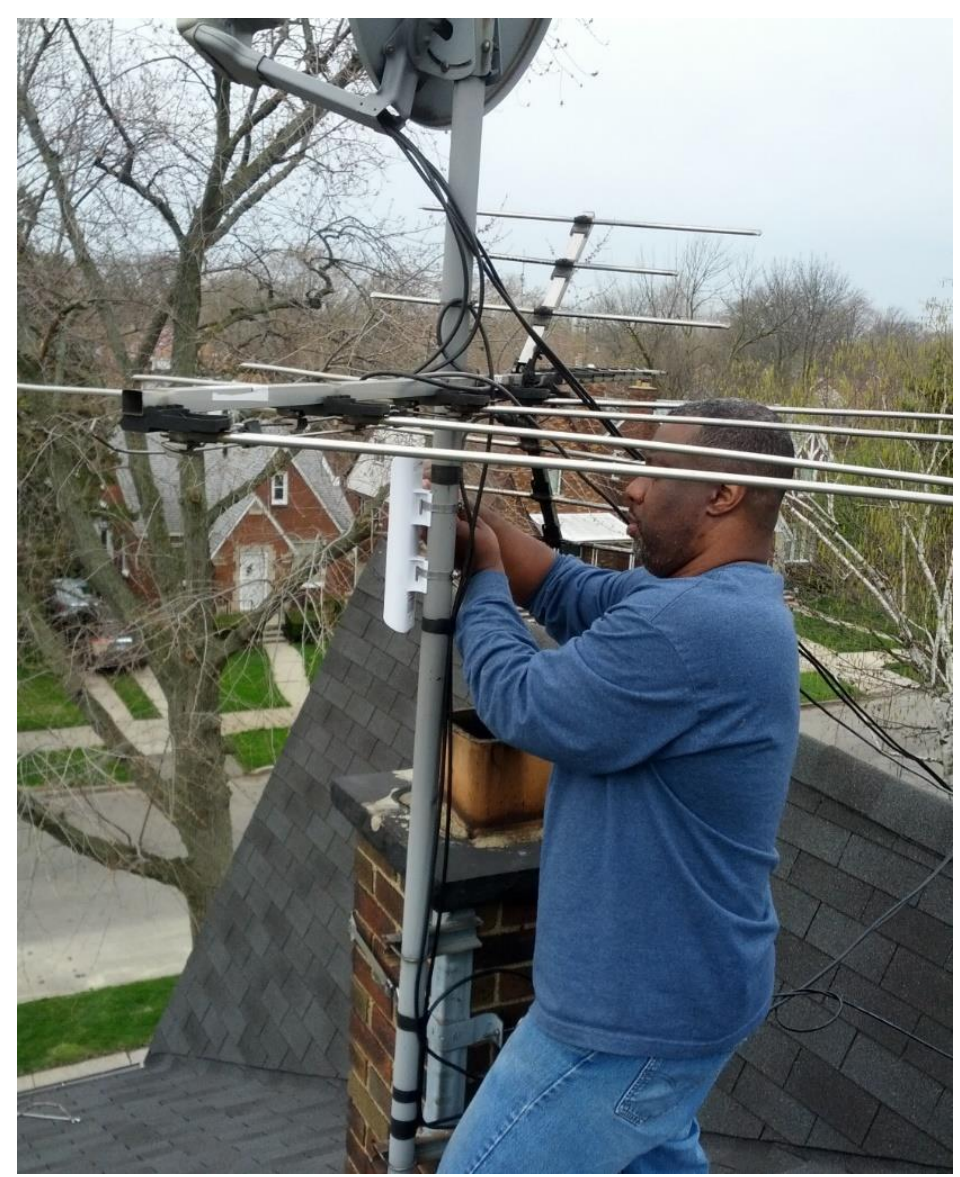

Segundo esses extratos, o caráter multicultural, operário e político da vila explica em parte seu caráter de laboratório tecnopolítico defensor da noção de justiça digital como meio de amenizar os efeitos da crise. Ver aí o modelo d'Alinsky talvez fizesse pular certos militantes originários de Motor City. Precisamos que nós falamos aqui de "técnicas" de organização que $A_{\text {linsky }}{ }^{20}$ professou ao longo de sua vida, não necessariamente de seu referencial político ${ }^{21}$. A novidade das ações comunitárias é de considerar a infraestrutura de informação criada e assegurada pelo conjunto de seus "leitores" - "espectadores" como um projeto participativo ("we are the networks, we are the media" declaram os membros de ECOSphere em uma entrevista). Se eles não tomam cuidados com os componentes da rede (antenas, alimentação elétrica, manutenção, etc.), se eles não se engajam para o bom funcionamento desses objetos, então a infraestrutura não funciona tão bem. Podemos, então, qualificar esses objetos como verdadeiras tecnologias participativas, de alavancas tecnológicas capazes de

${ }^{20}$ Sobre este ponto, ver ALINSKY, S. (1971), Rules for radicals : a practical primer for realistic radicals, Random House, New York.

${ }^{21}$ Referencial que insiste sobre a necessidade para os pobres de buscar seus interesses, mesmo "burgueses" ou "decadentes", o que não é o caso de Lee Boggs evocado anteriormente, nem mesmo das pessoas entrevistas aqui que se definem próximas do referencial político dos Black Panthers e do Black Power. 
implementar uma ideia do "care" ${ }^{22}$ e de infraestruturas frágeis que precisam de cuidado. As redes MESH fazem circular um saber e práticas culturais específicas de maneira decentralizada; elas nos parecem em relação com uma forma nova de estar junto que se desenvolve nesta vila "em crise".

\section{Da resiliência à rearticulação ${ }^{23}$, uma nova forma de comunidade}

Em 2011, Manuel Castells, estudando os cosmopolitismos múltiplos que são os movimentos da primavera árabe, os Idignados, Occupy e Anonymous, evocava novas formas de comunidade com conexões mais ou menos falhas, mas hiper-conectadas: “desta vez as redes digitais servem formas flexíveis e versáteis de organização e de debate, de grito de socorro, de distribuição de ideias e de iniciativas, de decisões coletivas distribuídas. As corajosas pessoas do movimento não estão nunca sozinhas, estão sempre conectadas, e, então, juntas não têm medo. A palavra de ordem delas: 'Todos juntos, nós podemos'. Poder o que? Para o momento, no discurso do movimento, o essencial é poder estar junto e, junto, descobrir uma outra democracia fazendo o caminho" (Op. Cit., 2011).

De nossa entrevista em Detroit, nós retemos agora que existe nos indivíduos com quem trabalhamos uma vontade de reconectar com um enfoque etnológico na política da cidade como uma forte determinação para engendrar uma dinâmica da mudança social vinda das ações de cidadãos e seu poder de agir. O importante é aqui também fazer junto, de engajar a "comunidade" para um projeto mais vasto para a cidade, de educá-la de maneira que as diferentes infraestruturas funcionem, nos papéis que um usuário pode ocupar. "A ambição" das implementações de rede MESH em Detroit é olhar para esse lado, na criação de novas comunidades.

Entrevistado: [...] Nós construímos isto de maneira horizontal agora, enquanto nós falamos. Você sabe, isto lembra muito ao que nós somos, a nossas ideias [...] a gente se dá conta hoje que nós não somos originais, que a gente pode se juntar a pessoas que partilham nossa visão de coisas nesta cidade. É isto que nós construímos no fundo, é disso que se trata, é isto que te trouxe aqui, você e sua tese sobre as infraestruturas decentralizadas $e$ horizontais...

\footnotetext{
22 "Cuidar, prestar atenção".

${ }^{23}$ Por "rearticulação", e seguindo Edgar Morin (2004), nós entendemos um ato pelo qual o indivíduo se conecta aos outros. Morin qualifica efetivamente este ato individual de "rearticulação". Ele utiliza o conceito de rearticulação em um sentido ativo que coloca em evidência a conexão viva entre o "bempensar" e o "bem-agir": "O pensamento complexo é o pensamento que reconecta. A ética complexa é a ética de reliance".
} 
nossa intenção nesse projeto era apresentar, era interagir com as outras organizações que dividem a mesma ideologia que nós, depois ver o que isto pode dar se trabalhamos todos juntos a partir da base.

Entrevistador: Na cidade invertida?

Entrevistado: É isto? Bem-vindo à cidade invertida!

O desenvolvimento de uma cidadania ativa e participativa através dessas redes comunitárias sem fio é um fato na cidade em crise que é Detroit, nós verificamos passando três meses ao lado desses Digital Stewards, associações que as treinam e pessoas que desenvolvem esse programa de trabalho em rede e de rearticulação comunitária. Enquanto uma cidade duramente atingida pela falência e uma grande pobreza, Detroit parece se transformar em exemplo de cidade pós-industrial moderna “onde as falsas promessas de consumo e produção, sem limites aos preços da dignidade humana, e do sentido de comunidade foram examinadas minuciosamente e, finalmente, substituídas por fundações mais humanistas" (HANDELSMAN, 2011).

As "Communities", as diferenças associações de Educação Popular e esses ativistas das infraestruturas ocupam um papel nesse empreendimento de rehumanização das infraestruturas de telecomunicação, de agricultura, de transporte, de educação, de gestão do lixo, de consumo etc. As práticas de transformação fundadas sobre os mundos do digital e as redes comunitárias sem fio, observadas na cidade em crise de Detroit, ilustra um "viver Internet" diferente. Eles podem também ser considerados como provas do desenvolvimento da economia da contribuição (STIEGLES, 2009), vetor do "poder de agir” dos cidadãos usuários (PROULX, 2012), e necessário a uma emancipação cidadã. A etnografia das redes sem fio comunitárias, meios de resiliência face à crise de Detróit, permite, igualmente, confirmar o fato de que a concentração espacial das classes populares pode se tornar um suporte de emancipação pela "revolta e revolução" 24 . Detroit tem, desde então, sua própria mídia sustentada por sua própria infraestrutura (as redes MESH). Assim, esta cidade possibilita refletir sobre um sistema político mais horizontal, o que seria um primeiro tijolo coerente construído para o empreendimento que se nomeia em todos os lugares como "sair da crise" e "reengajar os cidadãos na ação cívica e política".

\footnotetext{
${ }^{24}$ CLERVAL, A. [17 de Outubro de 2013], L'Humanité.fr, "À Paris, le discours sur la mixité sociale a remplacé la lutte des classes" [http://www.humanite.fr/societe/anne-clerval-paris-le-discours-sur-lamixite-socia-551401].
} 
Na França, seria preciso, talvez, revisitar simplesmente tudo o que colocamos sob o termo de Educação Popular e dizer que o movimento do mesmo nome, enfraquecido pela ação governamental há numerosos anos, contém também as sementes de uma cidadania mais justa e mais equitativa. Uma cidadania mais apta a lidar com as crises quaisquer que elas sejam.

\section{Bibliografia}

AKRICH, M. «Comment décrire les objets techniques?», Techniques et Culture, vol. 9, p. 49-64, 1987.

ALINSKY, S. Rules for radicals: a practical primer for realistic radicals, Random House, New York, 1971.

BEAUDOUIN, V. «Prosumer », Communications, n. 89, p. 131-139, 2011.

CARDON, D. La démocratie Internet Promesses et limites, La République des idées - Seuil, Paris, 2010.

CASTELLS, M. The Information Age: Economy, society and culture, Blackwell, Oxford, 2010 [first edition 1996-1998].

CASTELLS, M. « Ni dieu ni maître: les réseaux », Leçon inaugurale de la Chaire Analyse interdisciplinaire de la société en réseaux, 28 juin 2011, Collège d'études mondiales, Fondation Maison des sciences de l'homme, Paris, 2011.

CLERVAL, A, L'Humanité.fr, “À Paris, le discours sur la mixité sociale a remplacé la lutte des classes", 17 octobre 2013. [http://www.humanite.fr/societe/anne-clerval-parisle-discours-sur-la-mixite-socia-551401].

COSME, C. Politique de la ville, l'expérience américaine, Centre d'analyse stratégique, la documentation Française, Paris, 2012.

DENIS, J. \& PONTILLE, D. Petite sociologie de la signalétique. Les coulisses des panneaux de métro, Presses de l'École des mines, coll «Sciences sociales», Paris, 2010.

DUMAZEDIER, JVers une civilisation du loisir ?, Seuil, Paris, 1962.

EGYEDI, T. AND MEHOS, D. Inverse infrastructures Disrupting Networks from Below, Edward Elgar, Northampton, 2012.

FEENBERG, A. "Les dix paradoxes de la technologie », in La Recherche et l'Innovation en France - FutuRIS 2011 (éd. Odile Jacob), Association nationale de la recherche et de la technologie, Paris. 2011.

FEENBERG, A. Repenser la technique, La Découverte M.A.U.S.S, Paris, 2004.

FLICHY, P. «Internet et le débat démocratique », Réseaux, n.150, p. 159-185, 2008.

GALlAGHER, J. Reimagining Detroit: Opportunities for Redefining an American City, Wayne State University Press, Detroit. 2010

GALlAGHER, J. Revolution Detroit, Strategies for Urban Reinvention, Wayne State University Press, Detroit, 2013. 
GENTÈS, A. \& HUGUET, F. « Les alternatives aux réseaux sociaux : l'architecture distribuée et le design de média », in Réseaux sociaux, culture politique et ingénierie des réseaux sociaux (dir. Stiegler, B.), IRI \{FYP\} éditions, Paris, 2012.

GERBAUDO, P. Tweets and the Streets, Social Media and Contemporary Activism, Pluto Press, London, 2012.

GOËTA, S. UrbaNews.fr, "Détroit n'est pas une silencieuse ville sauvage. [La critique, par Aaron Handelsman]", 1er juin 2012. [http://www.urbanews.fr/2012/06/01/21179detroit-nest-pas-une-silencieuse-ville-sauvage-la-critique-par-aaron-handelsman/\#.UioD gLwUVTU].

GONZALEZ-QUIJANO, Y. Arabités numériques Le printemps du Web arabe, Sindbad Actes Sud, Arles, 2012.

HOLSTON, J. «Spaces of Insurgent Citizenship», Cities and Citizenship, Duke University Press, Durham and London, 1999.

JEANNERET, Y. Y-a-t-il (vraiment) des technologies de l'information?, Presses Universitaires du Septentrion, Villeneuve d'Ascq, 2007.

KLEINE, R. Detroit Free Press, "How the State of Michigan helped bankrupt Detroit", 4 août 2013. [http://www.freep.com/article/20130804/OPINION05/308040048/detroitfinancial-crisis-state-of-michigan-causes-bankruptcy]

LEE-BOGGS, G. \& KURASHIGE, S. The Next American Revolution, Sustainable Activism for the Twenty-first Century, University of California Press, Berkeley 2011.

LOVELUCK, B. La liberté par I'information. Généalogie politique du libéralisme informationnel et des formes de l'auto-organisation sur internet, Thèse de doctorat (dir. Marcel Gauchet), EHESS, Paris 2012.

MORIN, E. La Méthode. Volule 6: Éthique, Seuil, Paris 2006.

MUSIANI, FNains sans géants, Architecture décentralisée et services Internet, Thèse de doctorat (dir. Cécile Méadel), Ecole nationale supérieure des mines de Paris 2012.

MUSIANI, F. Caring about the plumbing: On the importance of architectures in social studies of (peer-to-peer) technology, Peer Production Journal, 2012 http://hal.archivesouvertes.fr/view_by_stamp.php?\&halsid=sd01ountu02dq0r3f7thqbkd

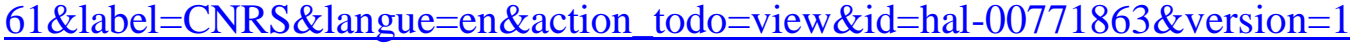

PLENEL, E. Mediapart.fr, "L'alarme d'Edgar Morin”. 25 août 2013 http://www.mediapart.fr/journal/culture-idees/240813/1-alarme-dedgarmorin?page_article=2

PROULX, S., L. HEATON, CHOON, M. J. K., AND MILLETTE, M. (), « Paradoxical empowerment of producers in the context of informational capitalism », New review of hypermedia and multimedia, vol. 1, n. 17, p. 9-29, 2011.

PROULX, S. « La puissance d'agir des citoyens à l'ère du numérique: cyberactivisme et nouvelles formes d'expression politique en ligne », in Le cyberactivisme au Maghreb et dans le Monde Arabe (dir. Najar, S.), Karthala, Paris, 2012.

PROULX, S. « Can the use of digital media favour citizen involvement », Global Media and Communication, vol. 3, n. 5, p. 293-307, 2009. 
STAR, S. L. « The Ethnography of Infrastructure », American Behavioral Scientist, vol. 3, n. 43, p. 377-391, 1999.

STAR, S. L. "Infrastructure and ethnographic practice: working on the fringes », Scandinavian Journal of Information Systems, vol. 2, n. 14, p. 107-122, 2002.

SUGRUE, T. The Origins of the Urban Crisis, Race and inequality in postwar Detroit, Princeton University Press, Princeton, 1996.

VANHEMERT, K. Wired, "The Best Map Ever Made of America's Racial Segregation, 26 août 2013. [http://www.wired.com/design/2013/08/how-segregated-isyour-city-this-eye-opening-map-shows-you\#slideid-210281].

WINNER, L. « Do artifacts have politics ? », The whale and the reactor : a search for limits in an age of high technology, University of Chicago Press, Chicago, p. 19-39. Anderson, C. (2012), Makers, The New Industrial Revolution, Crown, New York, 1986.

WOOLGAR, S. \& COOPER, G. « Do artefacts have ambivalence: Mose's bridges, Winner's bridges and other urban legends in S\&TS », Social Studies of Science, SAGE, Londres, p. 433-449, 1986. 


\section{Revista do Laboratório de}

Estudos Urbanos do Núcleo

de Desenvolvimento da Criatividade

\section{Para citar essa obra:}

HUGUET, François. 2. Por uma etnografia das redes sem fio comunitárias: implementar tecnologias decentralizadas para agir contra a crise em Detroit. In: RUA [online]. Especial - Cidade Conectada, p. 241-257 - ISSN 1413-2109/e-ISSN 2179-9911 - outubro/2016. Consultada no Portal Labeurb - Revista do Laboratório de Estudos Urbanos do Núcleo de Desenvolvimento da Criatividade.

http://www.labeurb.unicamp.br/rua/

Capa: The Best Map Ever Made of America's Racial Segregation.

Laboratório de Estudos Urbanos - LABEURB

Núcleo de Desenvolvimento da Criatividade - NUDECRI

Universidade Estadual de Campinas - UNICAMP

http://www.labeurb.unicamp.br/

Endereço:

LABEURB - LABORATÓRIO DE ESTUDOS URBANOS

UNICAMP/COCEN / NUDECRI

CAIXA POSTAL 6166

Campinas/SP - Brasil

CEP 13083-892

Fone/ Fax: (19) 3521-7900

Contato: http://www.labeurb.unicamp.br/contato 